\title{
Closed Loop Tuning of Cascade Controllers Based on Setpoint Experiment
}

\author{
Mohammad Atif Siddiqui*, MN Anwar**, SH Laskar***, M Shamsuzzoha**** and MR Mahboob***** \\ *Department of EIE, National Institute of Technology Silchar-788010, India \\ **Department of EE, National Institute of Technology Patna-800005, India \\ ***Department of EIE, National Institute of Technology Silchar-788010, India \\ ****Department of Chemical Engineering, KFUPM, Dhahran 31261, Saudi Arabia \\ *****Department of EE, Tezpur University, Sonitpur, Assam-784028, India \\ *Corresponding Author: atif@rs.ei.student.nits.ac.in
}

Submitted: 02/08/2019

Revised: 28/01/2020

Accepted: 06/02/2020

\begin{abstract}
This study presents online PI/PID controller tuning rules for cascade control configuration. The necessary process data are determined with the help of a simple setpoint experiment in a single closed-loop mode with the proportional controller only. The obtained process data is recorded in terms of the overshoot, controller gain, peak time, and relative output change. The data is then utilized to establish a correlation with PI/PID settings through simulations. Further, the proposed PI/PID controller tuning rule for a single loop has been extended to the cascade control configuration. The inner loop controller is tuned first, and then the primary loop is tuned by considering a well-tuned inner loop as a part of the primary plant. Finally, simulation examples demonstrate that the proposed method delivers significant disturbance rejection and better setpoint response when compared to the recently reported methods from the literature. The proposed method is also able to deliver stable closed-loop performances when subjected to large parametric uncertainties and measurement noise.
\end{abstract}

Keywords: Cascade control structure; PI/PID controller; Setpoint experiment.

\section{INTRODUCTION}

Proportional integral and derivative (PID) controllers are mostly utilized in the process industry due to their ease of applicability and flexibility. In the literature, numerous PI/PID controller tuning approaches are available (Luyben, 1989; Lee et al., 2002; Chen \& Seborg, 2002) that utilize either open-loop plant test or closed-loop plant test. The majority of the design methods are reported on the open-loop plant model (Rivera et al., 1986; Chen \& Seborg, 2002; Mellichamp et al., 2010; Anwar et al., 2019; Badgwell et al., 2019) and, therefore, require a two-fold procedure. The plant parameters are first recognized using the system identification method and experimental data. Subsequently, the parameters of the PI/PID controller are obtained by using a suitable design technique. For model identification, a step or pulse test is carried out to find plant data. This may make the model identification process sluggish, trouble the process, or even make the process out of control (Shamsuzzoha, 2013; Jeng, 2015). The closed-loop experiment is a viable solution to the drawbacks of the open-loop test that can be utilized to execute the online tuning of controllers. The classical method of Ziegler-Nichols (Z-N) (Ziegler \& Nichols, 1995) is an illustration of a closed-loop test experiment, but with few drawbacks such as

i) For lag/delay dominant process setting is aggressive/sluggish, which results in poor robustness.

ii) It cannot be used on second-order processes. 
iii) The closed-loop system needs to be brought to its verge of instability to obtain the process data, and this may require several trials.

In Tyreus \& Luyben (1992), the authors avoid the first shortcoming of the Z-N method by devising less aggressive PI settings over the lag dominant process. However, when the same settings are employed over the dominant process, the response becomes sluggish. The second shortcoming of the Z-N method is overcome by Luyben (2001), by extracting more information about the process through a modified relay feedback method. This method, however, fails for processes that are unstable or belong to inverse response characteristic.

Recently, several closed-loop test experiment based controller tuning approaches have been proposed to alleviate the shortcoming of the Z-N method (Shamsuzzoha, 2013; Jeng, 2015; Jeng \& Ge, 2016; Zeng et al., 2019). The aforementioned literature has addressed the closed-loop test experiment based tuning of the controller for unity feedback systems, but using closed-loop test experiment based design methods for the cascade control system (CCS) has received less attention. As the CCS comprises the design of two controllers, the tuning of CCS is more complex than the tuning of a simple feedback system. The rationale backing this complex CCS is that it produces good disturbance rejection as compared to the single loop configuration. With the aim to furnish users with efficient support in the tuning of the CCS, a closed-loop experiment based design method has been reported in Leva \& Marinelli (2009) and Veronesi \& Visioli (2011). Leva \& Marinelli (2009) gave a brief review of various autotuning methods for CCS and suggested a single step, single relay test, and two-step test approaches to design controller of CCS. Veronesi \& Visioli (2011) have proposed an automatic tuning method for PID controller in series with a first-order lag filter for low-order cascade processes. However, their method demands information about the existing controller. Later on, Jeng \& Lee (2012) proposed tuning rules for cascade control structure in which an apparent model rationalizes for secondary loop dynamics. But this model results in modelling errors due to model reduction. The above method shows superiority over the method of Veronesi \& Visioli (2011). In Jeng (2014), the author has improved the control performances of the cascade control system by proposing a control technique that utilizes process input and output data. This approach is restrictive in nature and tedious and may not work if the closed-loop data contains the effect of disturbance. Recently, Santosh \& Chidambaram (2019) have proposed a relay autotuning approach for open-loop unstable CCS. They have achieved improvement in servo and regulatory performance, but with a limitation. The strategy is applicable to unstable processes having time delay to time constant ratio less than 1 (Venkatashanker \& Chidambaram, 1994).

Further design approaches considered sophisticated CCS (Uma et al., 2010; Padhan \& Majhi, 2013; Dasari et al., 2016; Raja \& Ali, 2018; Yin et al., 2019) to control stable, integrating, and unstable process but in these literatures, there is no evidence how the design method can depend on closed-loop experiment data.

In this work, an attempt is made to control unstable first order plus time delay (UFOPDT) process, higherorder integrating plus time delay (IPTD), second-order plus dead time (SOPDT), and higher-order stable process with/without zero by using closed-loop experiment data. Therefore, this article focuses on the tuning of the simple controllers for CCS in a closed-loop mode. The closed-loop test data are obtained by performing the closed-loop test experiment with the proportional controller only. A setpoint change is made to obtain the setpoint response data. Once the process data is obtained, PI/PID controller settings are achieved by deriving a correlation between PI/ PID controller and setpoint response data. Because the acquired process data encloses straightforward and valuable information on process dynamics compared with mathematical process models achieved by system identification, approaches for controller design are anticipated to deliver an effective controller. By using the proposed scheme, the secondary controller and primary controller of the CCS are estimated. The secondary loop controller is designed first by utilizing the setpoint step test in a closed-loop mode. Once the secondary loop is tuned, the primary controller is then designed by performing a closed-loop step test on the complete primary loop considering a well-tuned inner loop as a part of the primary plant.

The present work focuses on the following:

1 Secondary and primary processes are identified based on closed-loop test with the proportional controller only. 
2 Cascade controllers are tuned by establishing a correlation between the PID controller and closed-loop setpoint response data.

3 The efficacy of the proposed method is demonstrated through the simulation of several examples taken from the literature.

In this manuscript, the cascade control configuration is presented. Then, the PI/PID controller design is explored. The design of the secondary and primary controller is described. Robustness analysis is performed. Afterwards, simulation and discussion are presented. Finally, the conclusion is given.

\section{CASCADE CONTROL CONFIGURATION}

The general cascade control structure shown in Figure 1 has been considered. In this cascade control structure, $G_{c 2}$ and $G_{c 1}$ represent the secondary and primary controllers, whereas $G_{p 2}$ and $G_{p l}$ denote the secondary and primary plants, respectively. $R_{1}$ is the setpoint command of the primary loop. $Y_{1}$ and $Y_{2}$ are the outputs of the primary and secondary loops, respectively. The output of the primary controller $R_{2}$ serves as the setpoint for the secondary loop. $U_{2}$ is the secondary controller output. $D_{1}$ and $D_{2}$ are the disturbances entering into the system.

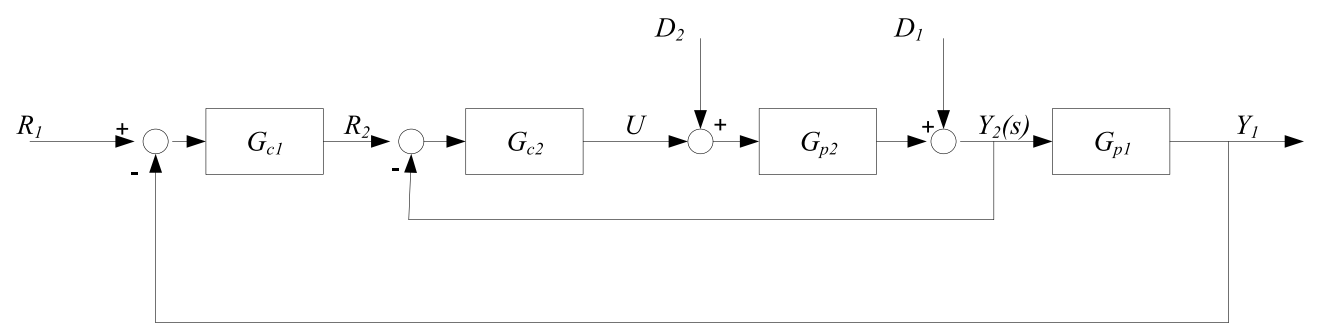

Figure 1. Block diagram of cascade control configuration.

In the cascade control configuration, the transfer function for the setpoint response of the secondary loop and the primary loop is given by Equations (1) and (2), respectively.

$$
\begin{aligned}
& \frac{Y_{2}}{R_{2}}=\frac{G_{c 2} G_{p 2}}{1+G_{p 2} G_{c 2}} \\
& \frac{Y_{1}}{R_{1}}=\frac{G_{c 1} G_{c 2} G_{p 1} G_{p 2}}{1+G_{p 2} G_{c 2}+G_{c 1} G_{c 2} G_{p 1} G_{p 2}}
\end{aligned}
$$

The controllers $G_{c 2}$ and $G_{c l}$ are considered as PI and PID controllers with the following transfer function:

$$
\begin{aligned}
G_{c 2} & =K_{c 2}+\frac{K_{i 2}}{s} \\
G_{c 1} & =K_{c 1}+\frac{K_{i 1}}{s}+K_{d 1} s
\end{aligned}
$$

where $K_{c 1}$ and $K_{c 2}$ are proportional constants, $K_{i 1}$ and $K_{i 2}$ are the integral constants, and $K_{d 1}$ is the derivative constant of the controllers. The parallel forms $G_{c 2}$ and $G_{c l}$ are used in this work because they decouple the proportional, integral, and derivative gains. Since the gains are decoupled, complexity in finding the gains from the designed method is reduced. In the Simulink software, the PID controller is usually set to ideal or parallel form along with the derivative filter coefficient $(\mathrm{N}) . \mathrm{N}$ is taken as 10, as it is a general practice in industry (Astrom \& Hagglund, 1995). A variant of the PID controller, namely, series form, is also commonly used. The PID structure for one arrangement can simply be transformed into other arrangements through mathematical formulas. 
The transfer function for a disturbance response of the secondary loop due to load $D_{2}$ is given by

$$
\frac{Y_{1}}{D_{2}}=\frac{G_{p 1} G_{p 2}}{1+G_{c 1} G_{c 2} G_{p 2} G_{p 1}+G_{p 2} G_{c 2}}
$$

Similarly, the transfer function for disturbance response for a primary loop due to load $D_{1}$ is given by

$$
\frac{Y_{1}}{D_{1}}=\frac{G_{p 1}\left(1+G_{c 2} G_{p 2}\right)}{1+G_{c 1} G_{c 2} G_{p 1} G_{p 2}+G_{c 2} G_{p 2}}
$$

\section{DESIGN OF THE SECONDARY AND PRIMARY CONTROLLER}

A cascade control structure is shown in Figure 1, where the secondary loop is nested inside the primary loop. The output $\left(R_{2}\right)$ of the primary loop controller acts as the setpoint for the secondary loop. In this work, the secondary loop is tuned first, and then the primary loop is tuned accordingly.

\section{Tuning of the secondary loop controller}

Consider the secondary loop structure of Figure 1, where the controller $G_{c 2}$ is assumed as a PI controller and is given by Equation (3). The secondary loop tuning involves two steps: first, the outer loop is disconnected from the inner loop, then the closed-loop setpoint experiment is performed by utilizing the controller in proportional mode only, i.e., switching off the derivate mode and integral mode. With the controller in P-mode, the proportional gain $\left(K_{c o}\right)$ value is varied until the overshoot of approximately $30 \%$ is achieved (Shamsuzzoha, 2013). The corresponding response obtained by the closed-loop setpoint experiment is shown in Figure 2.

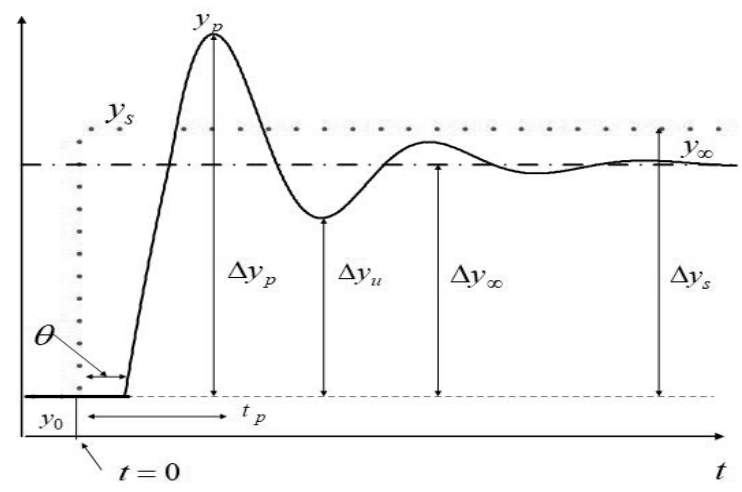

Figure 2. The response obtained with the P-only controller by the closed-loop setpoint experiment.

The following set of information has been extracted: (i) controller gain $\left(K_{c o}\right)$, (ii) overshoot, (iii) the time taken to reach the peak output $\left(t_{p}\right)$, and (iv) the relative steady state output change. Moreover, setpoint change, peak value change, and steady-state output change are also observed.

The experiment has been stopped as soon as the setpoint reached the first minimum in order to find $\Delta y_{\infty}$. This is normally being done to avoid time consumption (Shamsuzzoha \& Skogestad, 2010b; Shamsuzzoha, 2013). The corresponding output $\Delta y_{u}$ is recorded because $\Delta y_{\infty}$ value always lies in between the peak value $\left(\Delta y_{p}\right)$ and the first undershoot $\left(\Delta y_{u}\right)$ (Shamsuzzoha \& Skogestad, 2010).

$$
\Delta y_{\infty}=0.45 *\left(\Delta y_{p}+\Delta y_{u}\right)
$$

Once the process data is obtained, a relationship between the process data and the PI/PID controller needs to be established. In this work, IMC-PID (Seborg et al., 1990) rule modified similar to SIMC-rules (Skogestad, 2004) has 
been used to derive the PI/PID controller. For FOPDT (First-order plus time delay) processes, simplified IMC-PID tuning rule similar to SIMC rule is obtained by considering $\tau_{c}=\theta$ as

$$
\begin{aligned}
K_{c} & =\frac{2 \tau+\theta}{3 k \theta} \\
\tau_{i} & =\min \left\{\left(\tau+\frac{\theta}{2}\right), 8 \theta\right\} \\
\tau_{d} & =\frac{\tau \theta}{2 \tau+\theta}
\end{aligned}
$$

where $K_{c}$ is the proportional constant, $\tau_{i}$ is the integral constant, and $\tau_{d}$ is the derivative constant of the controller.

Now, the objective is to derive a relationship between the Equations (8)-(10) and the response data obtained from the step test experiment. To develop the relationship between the Equations and set point response data, 15 FOPDT processes are studied, which covers a broad range of processes by varying $\tau / \theta$ ratio such as $0.1,0.2,0.4,0.8,1.0,1.5$, $2.0,2.5,3.0,7.5,10.0,20.0,50.0$, and 100.0. The above ratios include a wide-ranging process, from delay-dominant to lag-dominant.

Six distinct proportional gain values are chosen to obtain closed-loop responses with the overshoot value of $0.1,0.2$, $0.3,0.4,0.5$, and 0.6 . In aggregate, 90 closed-loop experiments were conducted and for each of these responses, four data values like proportional gain $K_{c 0}$, overshoot, time to reach first overshoot $\mathrm{t}_{\mathrm{p}}$, and $b=\Delta y_{\infty} / \Delta y_{s}$ were recorded. With the help of Shamsuzzoha (2013), a simple correlation has been established between setpoint response experiment data and Equations (8)-(10). With the proportional gain as $K_{c 0}$ and overshoot as $M_{p}$, the PID controller obtained is given as

$$
\begin{aligned}
& K_{c}=K_{c o}\left(1.55 *(M p)^{2}-2.159 *(M p)+1.35\right) \\
& \tau_{i}=\min \left(0.645 * \delta *\left|\frac{b}{1-b}\right| * t_{p}, 2.44 * t_{p}\right) \\
& \tau_{d}=0.14 * t_{p} \quad \text { if } \quad \delta *\left|\frac{b}{1-b}\right| \geq 1 \\
& \text { Here, } \delta=1.55 *(M p)^{2}-2.159 *(M p)+1.35
\end{aligned}
$$

The proposed method has been derived by considering $\tau_{c}=\theta$; however, the less aggressive setting can be obtained by considering $\tau_{c}>\theta$ and fast response can be obtained by choosing $\tau_{c}<\theta$. In this regard, factor ' $\mathrm{F}$ ' has been incorporated ( $\mathrm{F}$ greater than 1 indicates setting that is less aggressive and factor $\mathrm{F}$ smaller than 1 indicates setting that is more aggressive). So, F has been incorporated in $K_{c}$ and $\tau_{i}$ and the final method is as follows:

$$
\begin{aligned}
& K_{c}=\frac{K_{c o}\left(1.55 *(M p)^{2}-2.159 *(M p)+1.35\right)}{F} \\
& \tau_{i}=F *\left(\min \left(0.645 * \delta *\left|\frac{b}{1-b}\right| * t_{p}, 2.44 * t_{p}\right)\right) \\
& \tau_{d}=0.14 * t_{p} \text { if } \delta *\left|\frac{b}{1-b}\right| \geq 1
\end{aligned}
$$

The "fast and robust" PI/PID settings can be obtained by considering F=1. 
Since the secondary loop is PI controller, $K_{c 2}$ and $K_{i 2}$ values are obtained by (i) setting the integral gain to zero, (ii) using the controller in P mode only, (iii) determining the overshoot, $t_{p}$ and $b$ from the response, (iv) using Equations (14) and (15), and (v) then representing the ideal form of PI controller into a parallel form of PI controller as $K_{c 2}=K_{c}$ and $K_{i 2}=K_{c 2} / \tau_{i}$.

\section{Tuning of the primary loop controller}

To account for the secondary loop dynamics, the primary loop controller is tuned by considering a well-tuned secondary loop as an integral part of the primary process. The closed-loop test is performed on the primary loop by switching the primary loop controller to P-mode only. Using closed-loop response obtained from the closed-loop test, the following set of information is achieved such as overshoot $\left(M_{p l}\right), b_{1}$ and $t_{p l}$. These values and Equations (8)-(16) are then utilized to achieve primary loop PID parameters as

$$
\begin{aligned}
& K_{c 1}=\frac{K_{c o 1}\left(1.55 *(M p 1)^{2}-2.159 *(M p 1)+1.35\right)}{F} \\
& K_{i 1}=K_{c 1} / F *\left(\min \left(0.645 * \delta_{1} *\left|\frac{b_{1}}{1-b_{1}}\right| * t_{p 1}, 2.44 * t_{p 1}\right)\right) \\
& K_{d 1}=K_{c 1}\left(0.14 * t_{p 1}\right) \text { if } \delta_{1} *\left|\frac{b_{1}}{1-b_{1}}\right| \geq 1
\end{aligned}
$$

By following the above procedure, one can obtain the primary loop controller settings.

\section{ROBUSTNESS ANALYSIS}

Proper robustness analysis is required for a control system having parametric uncertainty in their plant transfer function to achieve suitable closed-loop performance. These parametric uncertainties usually occur due to the lack of knowledge of the actual system. Plants with parametric uncertainty can be investigated for robustness analysis by (i) small gain theorem, (ii) maximum sensitivity to modelling error, and (iii) Kharitonov's theorem (Padhan \& Majhi, 2012). In this work, Kharitonov's theorem is utilized for robustness analysis. This theorem is a renowned tool to decide the stability of the interval polynomial having an infinite number of members (Barnish et al., 1989). It states that an interval polynomial having an infinite number of members is robustly stable if and only if each of the four Kharitonov's polynomials is Hurwitz stable. Hurwitz's stable condition is checked by Routh stability criteria, where all the roots of the four Kharitonov's polynomial must lie on the left half plane or have negative real parts. The theorems proposed by Barnish (1989) and Bhattacharyya \& Keel (1995) are the significant results of Kharitonov's theorem. The validation of the results is mainly established on the movement of the Kharitonov's rectangles in a counterclockwise direction about the origin and excluding the origin. Satisfying the above conditions means that closed-loop system is robustly stable.

Interval polynomial may be given as

$$
K(s)=q_{0}+q_{1} s+q_{2} s^{2}+q_{3} s^{3}+q_{4} s^{4}+q_{5} s^{5}+q_{6} s^{6} \ldots \ldots \ldots q_{i} s^{i}
$$

The four Kharitonov's polynomials are given by.

$$
\begin{aligned}
& k_{1}(s)=q_{0}^{\min }+q_{1}^{\min } s+q_{2}^{\max } s^{2}+q_{3}^{\max } s^{3}+q_{4}^{\min } s^{4}+\ldots \\
& k_{2}(s)=q_{0}^{\max }+q_{1}^{\max } s+q_{2}^{\min } s^{2}+q_{3}^{\min } s^{3}+q_{4}^{\max } s^{4}+\ldots
\end{aligned}
$$


$k_{3}(s)=q_{0}^{\max }+q_{1}^{\min } s+q_{2}^{\min } s^{2}+q_{3}^{\max } s^{3}+q_{4}^{\max } s^{4}+\ldots$

$k_{4}(s)=q_{0}^{\min }+q_{1}^{\max } s+q_{2}^{\max } s^{2}+q_{3}^{\min } s^{3}+q_{4}^{\min } s^{4}+\ldots$

where $q_{i}^{\min }$ and $q_{i}^{\max }$ represent the upper and lower bounds for the continuous set $q_{i}$.

To carry out robustness analysis, a CCS is considered with the secondary and primary plants (see Example-2 $\left(\right.$ Case-2)) as $G_{p 1}=\frac{1}{(10 s+1)(4 s+1)(s+1)^{2}}$ and $G_{p 2}=\frac{e^{-0.1 s}}{1.9 s+1}$, respectively.

The characteristic equation of the CCS is given by

$$
1+G_{p 2} G_{c 2}+G_{c 1} G_{c 2} G_{p 2} G_{p 1}=0
$$

It is presumed that the actual plant transfer function of the cascade structure is having $\pm 20 \%$ perturbation in time constants, gains, and time delays. With these perturbations, the four Kharitonov polynomials are obtained as follows:

$$
\begin{aligned}
& K_{1}(\mathrm{~s})=15.12+614.03 \mathrm{~s}+3318.99 \mathrm{~s}^{2}+3508.19 \mathrm{~s}^{3}+554.17 \mathrm{~s}^{4}+32.17 s^{5} \\
& K_{2}(\mathrm{~s})=18.14+1053.3 \mathrm{~s}+1700.57 \mathrm{~s}^{2}+1621.6 s^{3}+1272.4 s^{4}+59.92 s^{5} \\
& K_{3}(\mathrm{~s})=18.14+614.03 \mathrm{~s}+1700.57 \mathrm{~s}^{2}+3508.91 s^{3}+1272.4 s^{4}+32.17 s^{5} \\
& K_{4}(\mathrm{~s})=15.12+1053.29 \mathrm{~s}+3318.99 \mathrm{~s}^{2}+1621.59 \mathrm{~s}^{3}+554.17 s^{4}+59.92 s^{5}
\end{aligned}
$$

Table 1. The roots of Kharitonov polynomials.

\begin{tabular}{cccc}
\hline$K_{1}(\mathrm{~s})$ & $K_{2}(\mathrm{~s})$ & $K_{3}(\mathrm{~s})$ & $K_{4}(\mathrm{~s})$ \\
\hline$-8.06+5.11 \mathrm{i}$ & -19.94 & -36.6 & -6.26 \\
$-8.06-5.11 \mathrm{i}$ & $-0.21+0.98 \mathrm{i}$ & -2.44 & $-1.30+2.38 \mathrm{i}$ \\
-0.86 & $-0.21-0.98 \mathrm{i}$ & $-0.23+0.37 \mathrm{i}$ & $-1.30+2.38 \mathrm{i}$ \\
-0.21 & -0.85 & $-0.23-0.37 \mathrm{i}$ & -0.36 \\
-0.03 & -0.017 & -0.03 & -0.015 \\
\hline
\end{tabular}

The roots of Equations (26)-(29) are shown in Table 1, and it is observed that all the roots of the Kharitonov polynomial have a negative real part. Thus, Hurwitz's stability condition is satisfied. Kharitonov rectangles of the closed-loop system are generated by utilizing Equations (26)-(29) and varying the frequency from 0 to $1.2 \mathrm{~Hz}$ with an interval of 0.01 in Matlab. 


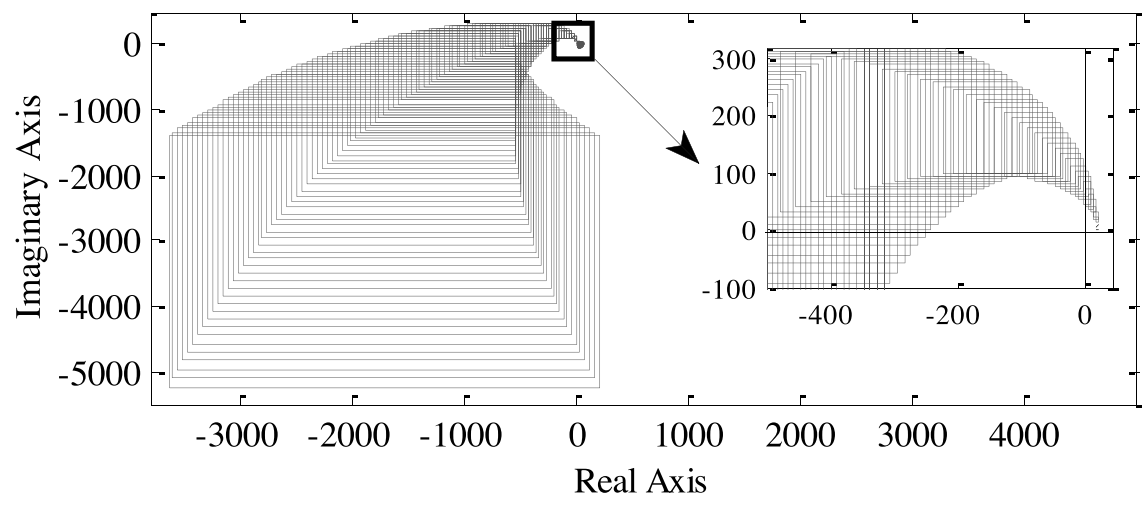

Figure 3. Kharitonov's rectangles obtained by the proposed method for Example-1.

The Kharitonov rectangles obtained are shown in Figure 3, and it is seen in the complex plane that Kharitonov rectangles move in a counterclockwise direction about the origin, excluding the origin. Satisfying the zero exclusion principle, thus, it is concluded that the closed-loop control system is stable and the controller is robust.

\section{SIMULATION RESULTS}

The performance of the proposed tuning approach has been compared with several case studies as reported in the literature. Different types of primary and secondary processes have been opted to establish the effectiveness of the proposed approach. The primary process is considered as higher-order stable process with/without zero, second-order plus dead time (SOPDT), higher-order integrating plus time delay (IPTD), and unstable first-order plus time delay (UFOPDT). The secondary process comprises FOPDT plus higher order. Simulations are carried out by considering these complex primary and secondary process and are compared with recently published works (Alfaro et al., 2009; Jeng, 2014; Azar \& Serrano, 2014; Kaya \& Nalbantoglu, 2016; Santosh \& Chidambaram, 2019).

Quantitative analysis of the output has been done on the basis of i) peak value of the system output $\left(y_{\max }=\max \left|Y_{1}\right|\right)$ and ii) integral absolute error $\left(I A E=\int_{0}^{\infty}|r(t)-y(t)| d t\right)$ and has been compared with the other reported methods. Better performance of the method is indicated by a lesser value of $\mathrm{y}_{\max }$ and $I A E$. The performance measures are listed in Table 2. In the practical world, noise emerges from the process itself, control valves, and measurement devices. Therefore, to justify the effectiveness of the present scheme under noisy situations, simulations of examples are conducted by considering the effect of white noise having noise power of 0.0001 , seed equal to 0 , and sampling time of $0.5 \mathrm{~s}$ on the process output. Once the effectiveness of the present approach is demonstrated under noisy conditions, robustness analysis is carried out by introducing perturbation in either primary or secondary or both the primary and secondary plants to achieve worst case model mismatch. To demonstrate the effectiveness of the present approach, five examples for simulation are considered as follows.

\section{Example-1}

In this example, the following primary and secondary processes are taken from the recent literature (Kaya \& Nalbantoglu, 2016) as

$$
G_{p 1}=\frac{e^{-s}}{(s+1)^{2}} \text { and } G_{p 2}=\frac{e^{-0.1 s}}{0.1 s+1}
$$

The present tuning approach yields $G_{c 1}$ and $G_{c 2}$ settings as listed in Table 2 (example-1). The present strategy is compared with the strategy given by Kaya \& Nalbantoglu (2016). The servo response is evaluated by applying a unit 
step setpoint change at $\mathrm{t}=0 \mathrm{~s}$, and the regulatory response is generated by introducing a unit step load disturbance $d_{2}=1$ at $t=100 \mathrm{~s}$ and $d_{1}=1$ at $t=200 \mathrm{~s}$. The corresponding closed-loop response is shown in Figure 4. As it is seen in the figure, the methodology proposed by Kaya \& Nalbantoglu (2016) has large overshoot and long settling time in regulatory response. On the other hand, the proposed method has a smaller overshoot.

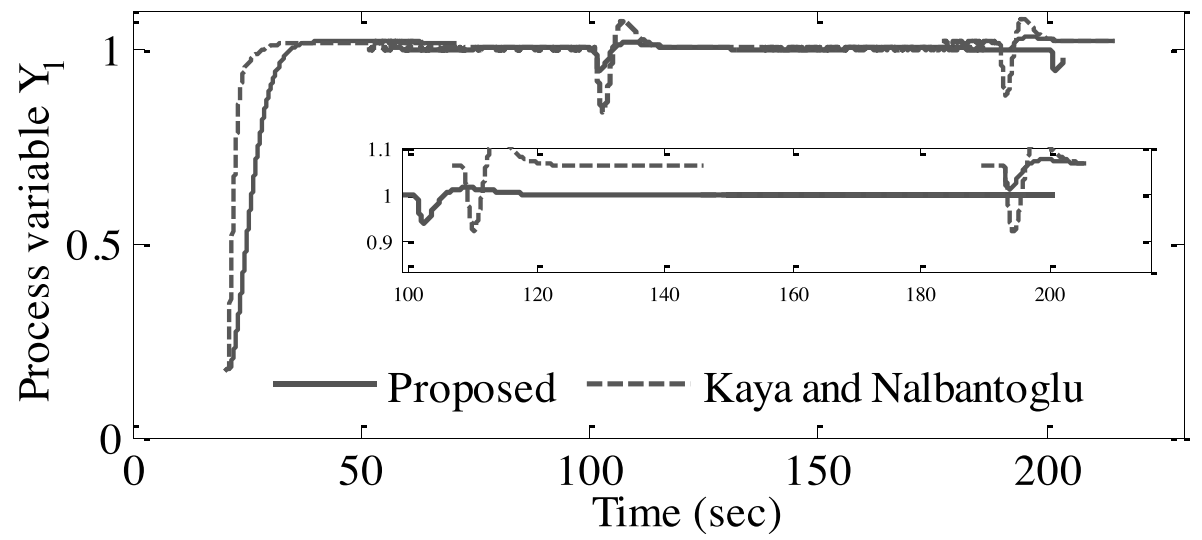

Figure 4. Process response for Example-1.

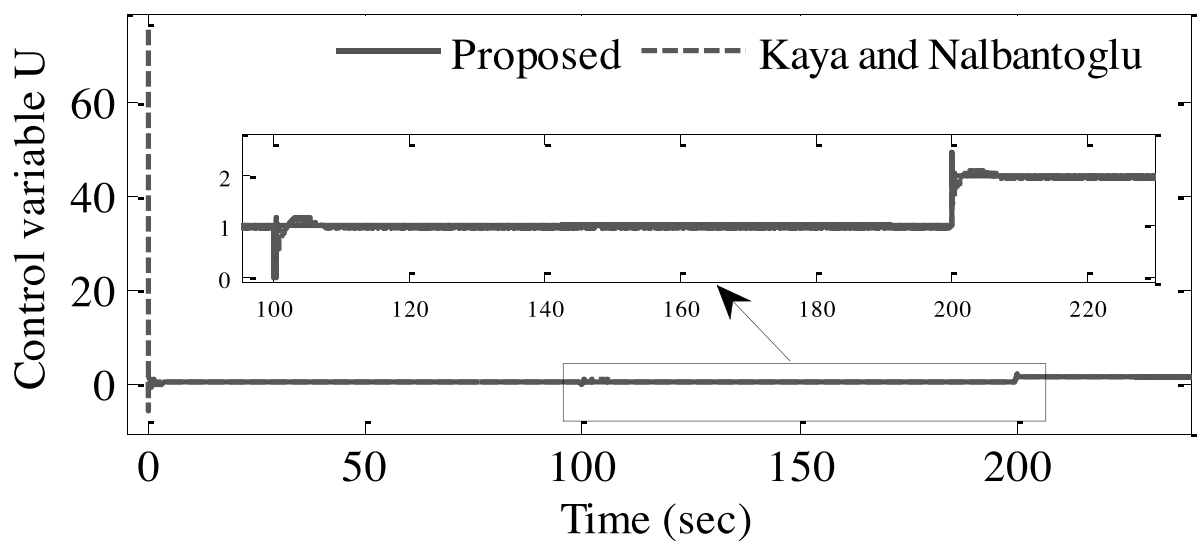

Figure 5. Controller response for Example-1.

Figure 5 shows controller efforts for Example-1. At first, the sharp hike in the controller response of the Kaya \& Nalbantoglu (2016) is observed, whereas the proposed method shows smooth controller efforts. In examples 2, 3, 4, and 5, smooth controller efforts have been found, though not shown. Figures 4 and 5 and the performance parameter tabulated in Table 2 (Example-1) show that the present strategy yields better and improved regulatory performance with lesser controller efforts compared to Kaya \& Nalbantoglu (2016). In a real-world scenario, disturbance rejection (regulatory performance) is more crucial than servo performance as the setpoint can be altered by using a setpoint filter or setpoint weighting. On the other hand, disturbance can occur at any point of time that cannot be altered by a setpoint filter or weighting factor. Figures and the performance parameter tabulated in Table 2 show that the present strategy yields better and improved regulatory performance. Hence, the present scheme is more appropriate for the process industry owing to its better disturbance rejection. To justify the effectiveness of the present scheme under noisy situations, simulations of examples are conducted by considering the effect of white noise. The resulting closedloop response and controller response are shown in Figures 6 and 7, respectively. 


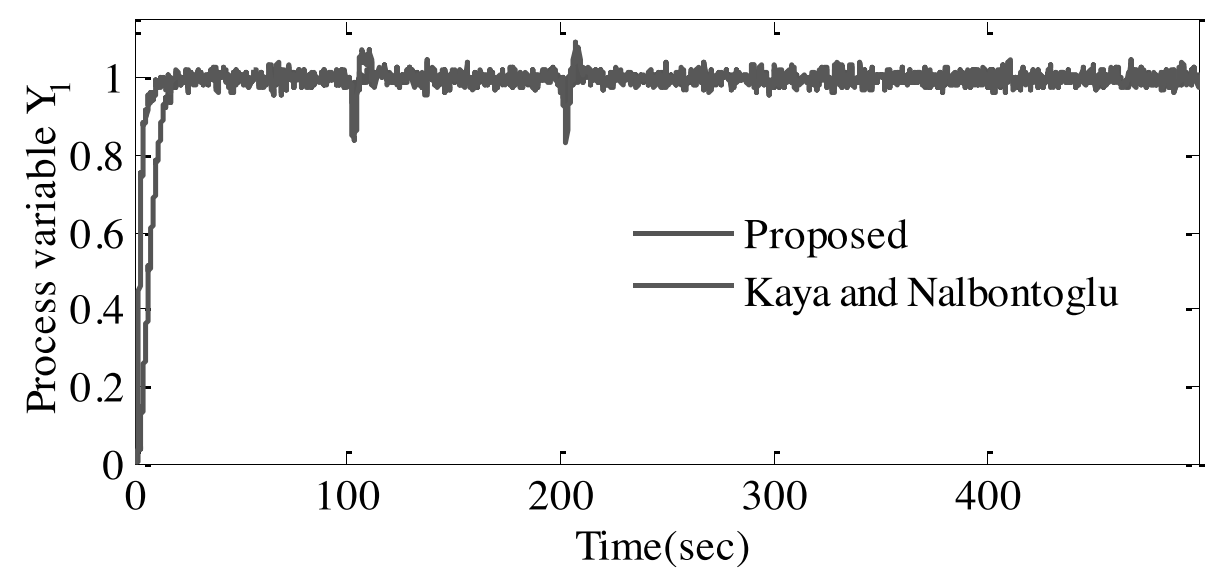

Figure 6. Process variable response due to noise for Example-1.

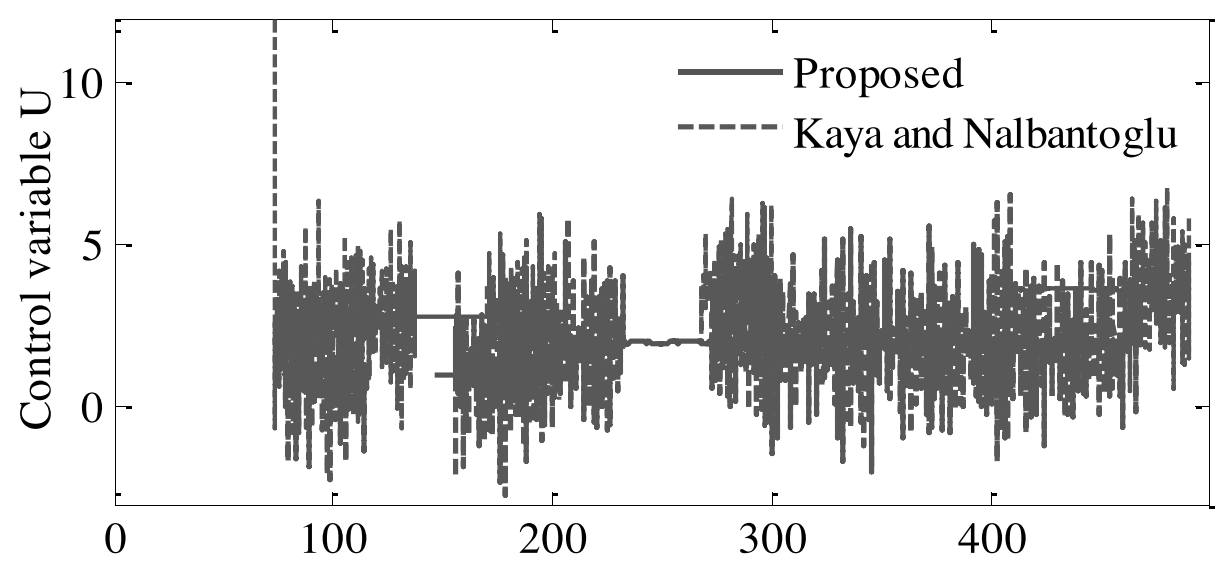

Figure 7. Controller response due to noise for Example-1.

As seen, the proposed approach can give a robust performance with smooth controller efforts under the effect of noise. With the intention to demonstrate the robustness of the proposed scheme, perturbations are introduced in $G_{p l}$ and $G_{p 2}$. Gain and time delay are perturbed by $+20 \%$, whereas time constant is perturbed by $-20 \%$ in both $G_{p 1}$ and $G_{p 2}$. It is to be noted from Figure 8 that the present scheme is able to give robust closed-loop performance.

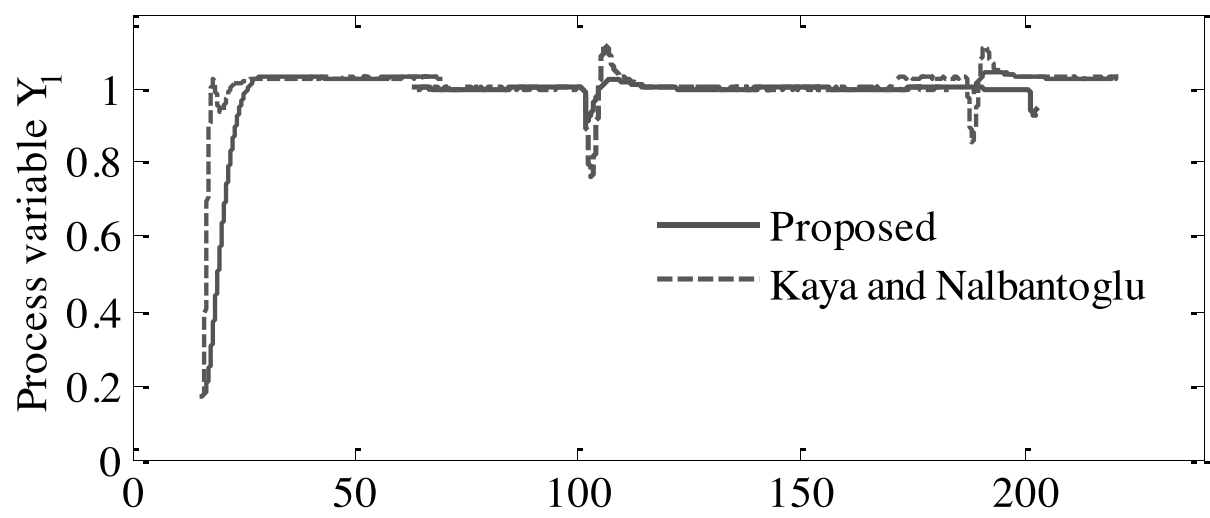

Figure 8. Perturbed response for Example-1. 


\section{Example-2}

The cascade structures studied by Jeng (2014) with three different cases are considered:

CASE-1

$G_{p 1}=\frac{1}{(10 s+1)(4 s+1)(s+1)^{2}}$ and $G_{p 2}=\frac{e^{-0.1 s}}{1.9 s+1}$

Here, the primary plant is of higher order, and the secondary plant is FOPDT.

$\underline{\text { CASE-2 }}$

$G_{p 1}=\frac{10(-5 s+1) e^{-5 s}}{(30 s+1)^{3}(10 s+1)^{2}}$ and $G_{p 2}=\frac{3 e^{-3 s}}{13.3 s+1}$

In this structure, the primary plant is of higher order and exhibits nonminimum phase dynamics. The secondary plant is FOPDT with large time delay and time constant.

\section{CASE-3}

$$
G_{p 1}=\frac{e^{-3 s}}{s(10 s+1)(2 s+1)^{2}} \text { and } G_{p 2}=\frac{e^{-0.5 s}}{s+1}
$$

Here, the secondary plant is FOPDT process. The primary loop plant is higher-order system with integrator and time delay. The control of such processes is difficult and challenging.

The controller settings obtained by the present tuning approach for Case-1-3 are listed in Table 2 (Example 2). For the purpose of comparison, the performance of the present scheme is compared with that of Jeng (2014). The servo response is evaluated by applying a unit step setpoint change at $\mathrm{t}=0 \mathrm{~s}$ for the three cases. The regulatory response is generated by introducing a unit step load disturbance as $d_{2}=1$ at $\mathrm{t}=0 \mathrm{~s}$ and $d_{1}=1$ at $\mathrm{t}=80 \mathrm{~s}$ for Case- $1, d_{2}=1$ at $\mathrm{t}=0 \mathrm{~s}$ and $d_{1}=1$ at $\mathrm{t}=750 \mathrm{~s}$ for Case $-2, d_{2}=1$ at $\mathrm{t}=0 \mathrm{~s}$ and $d_{1}=1$ at $\mathrm{t}=250 \mathrm{~s}$ for Case- 3 . The closed-loop response and noise response are shown in Figures 9-14.
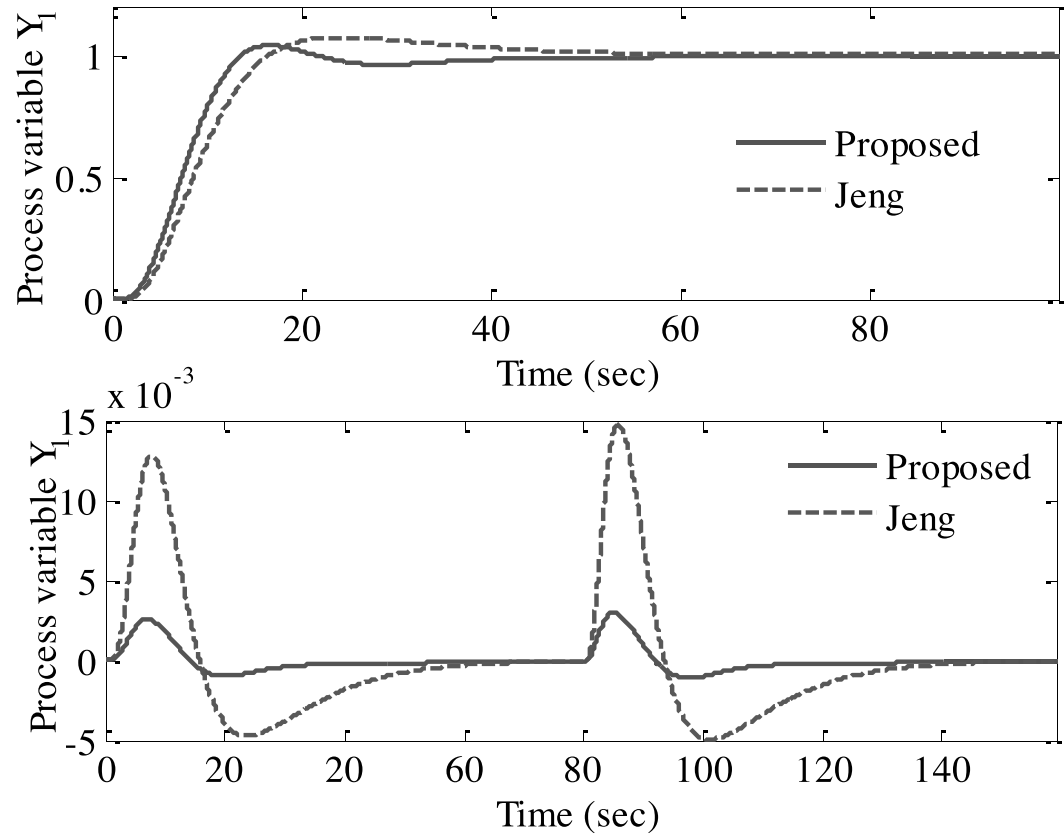

Figure 9. Servo response and regulatory response for Example-2 (Case-1). 

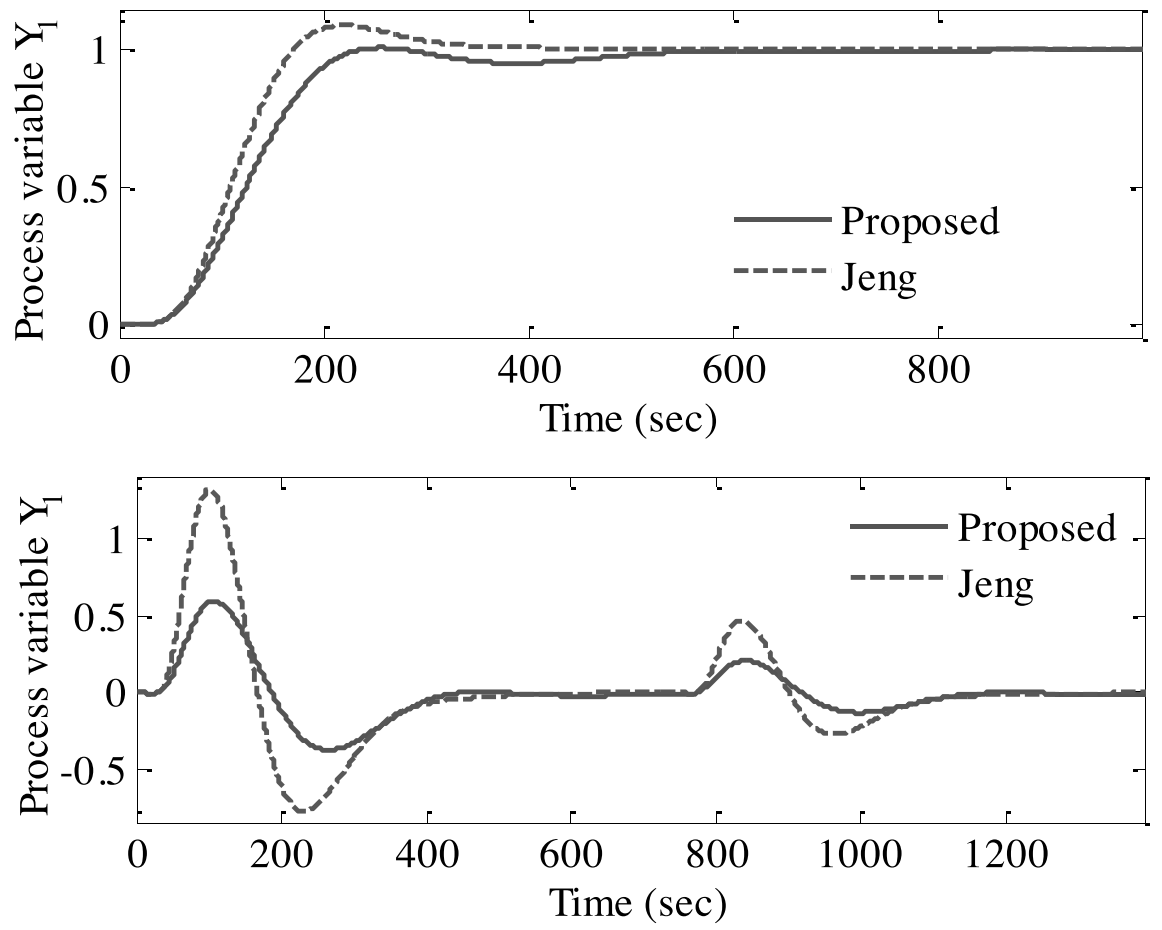

Figure 10. Servo response and regulatory response for Example-2 (Case-2).
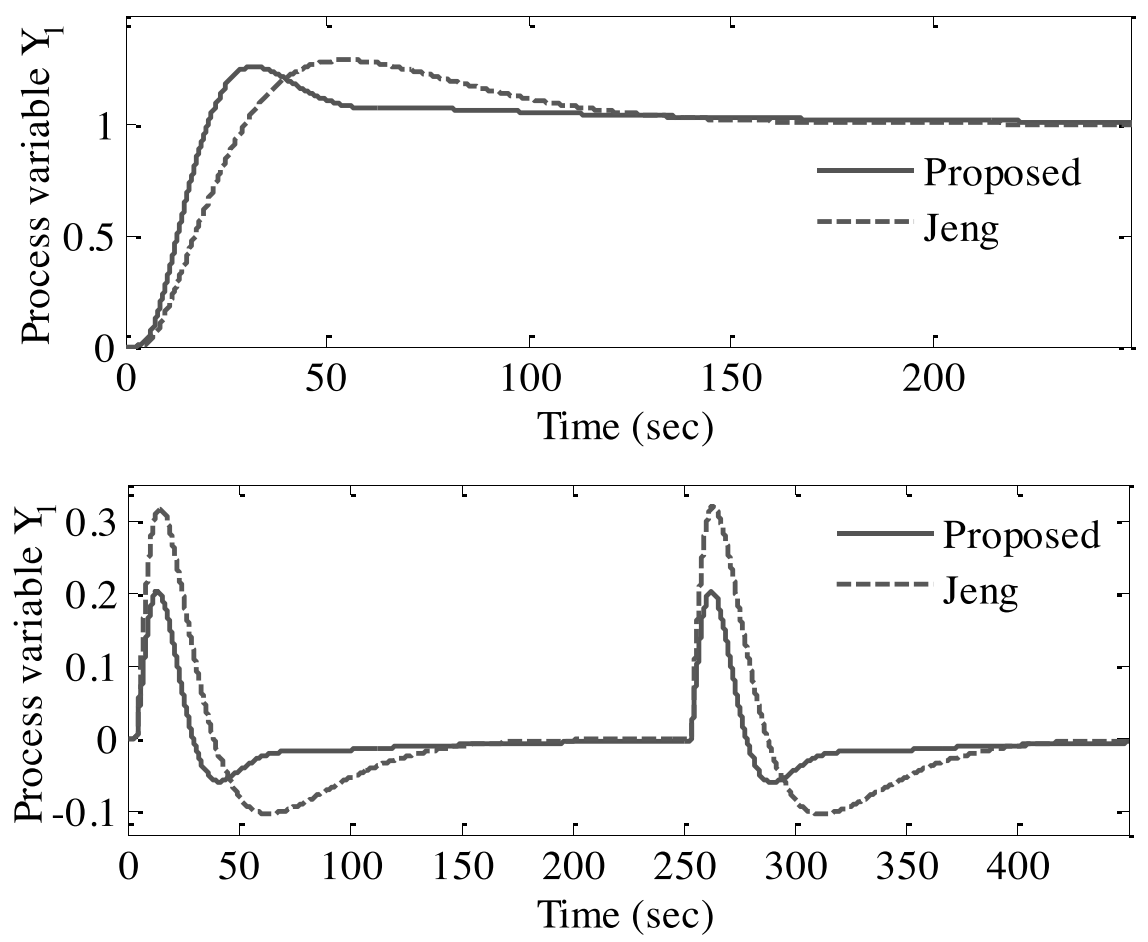

Figure 11. Servo response and regulatory response for Example-2 (Case-3). 


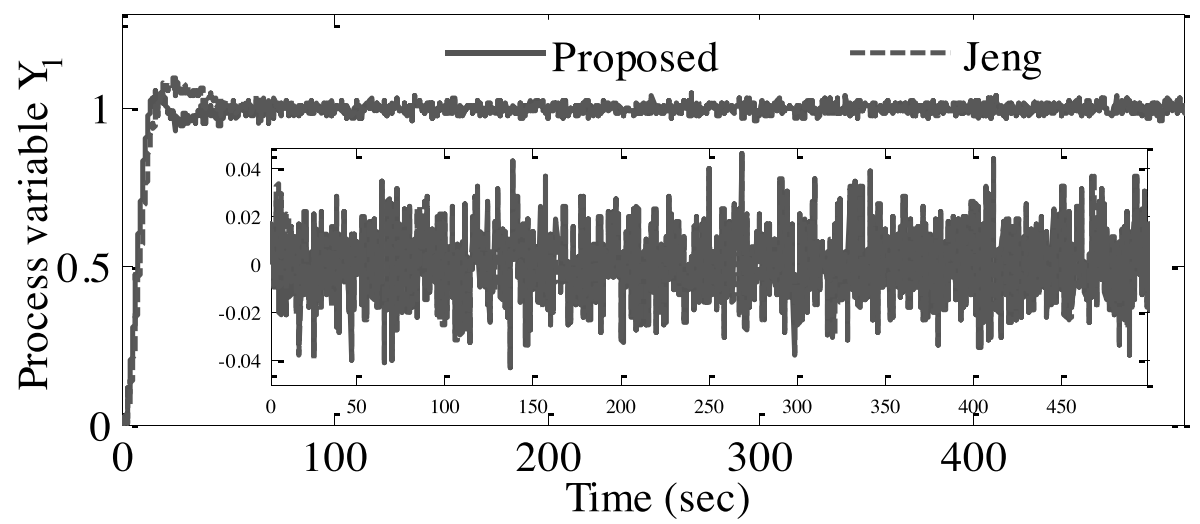

Figure 12. Process variable response due to noise for Example-2 (Case-1).

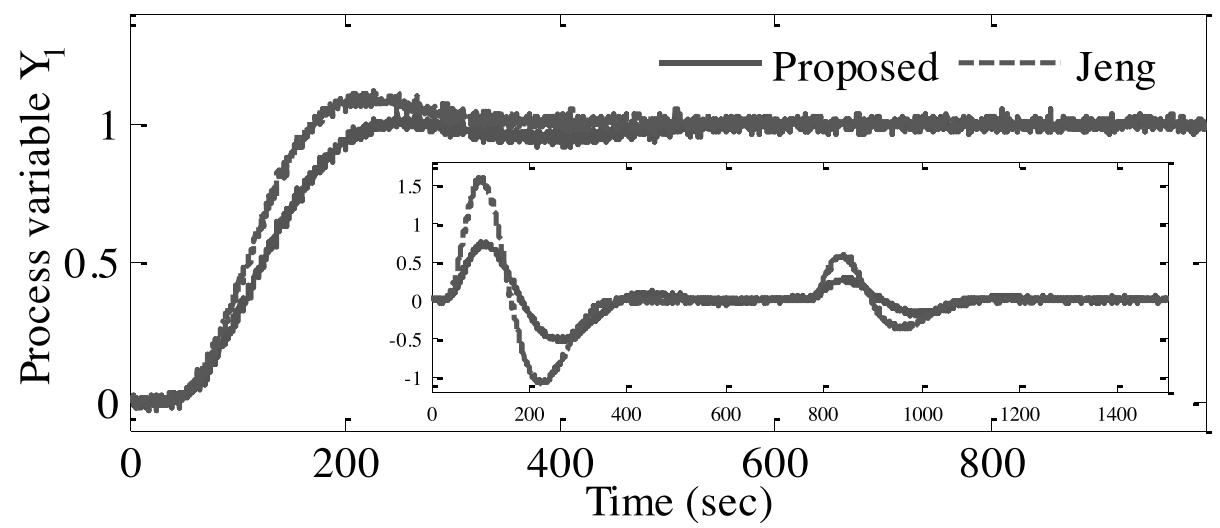

Figure 13. Process variable response due to noise for Example-2 (Case-2).

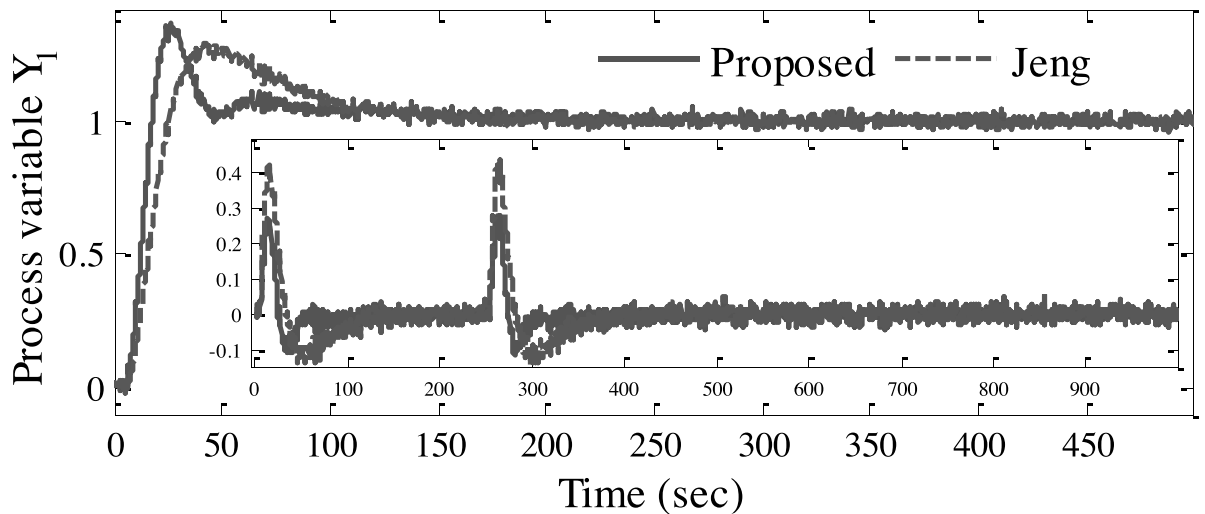

Figure 14. Process variable response due to noise for Example-2 (Case-3).

Figures 9-14 and the performance parameter tabulated in Table 2 (Example 2- Cases 1, 2, and 3) show that the present strategy has significant improvement in regulatory performance and has good robustness under noisy conditions. With the intention to demonstrate the robustness of the proposed scheme, perturbations are introduced in the process. For case-1, time delay and gain of $G_{p 2}$ are changed by $+30 \%$, whereas the gain of $G_{p 1}$ is changed by $+30 \%$. For case- 2 and case-3, gain and time delay of $G_{p 1}$ are changed by $+20 \%$. The perturbed response for cases 1,2 , and 3 is generated for load disturbance change and is shown in Figures 15-17, respectively. 


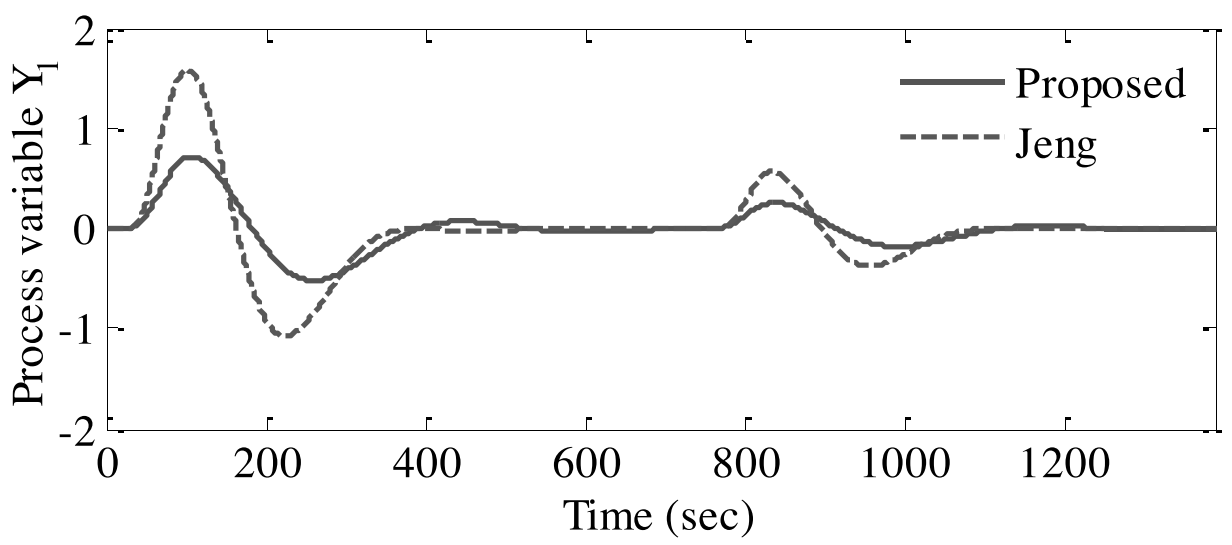

Figure 15. Perturbed response for Example-2 (Case-1).

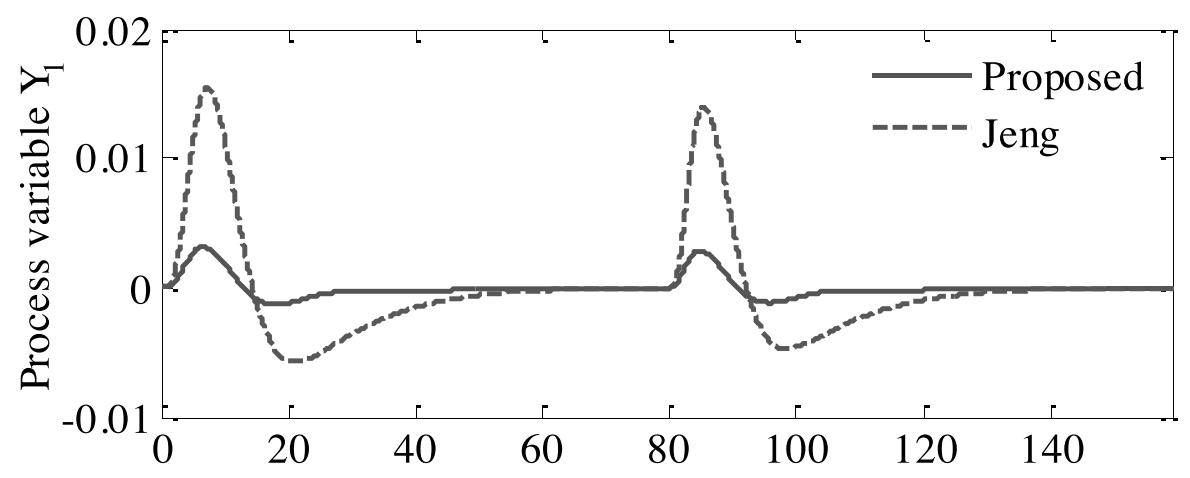

Figure 16. Perturbed response for Example-2 (Case-2).

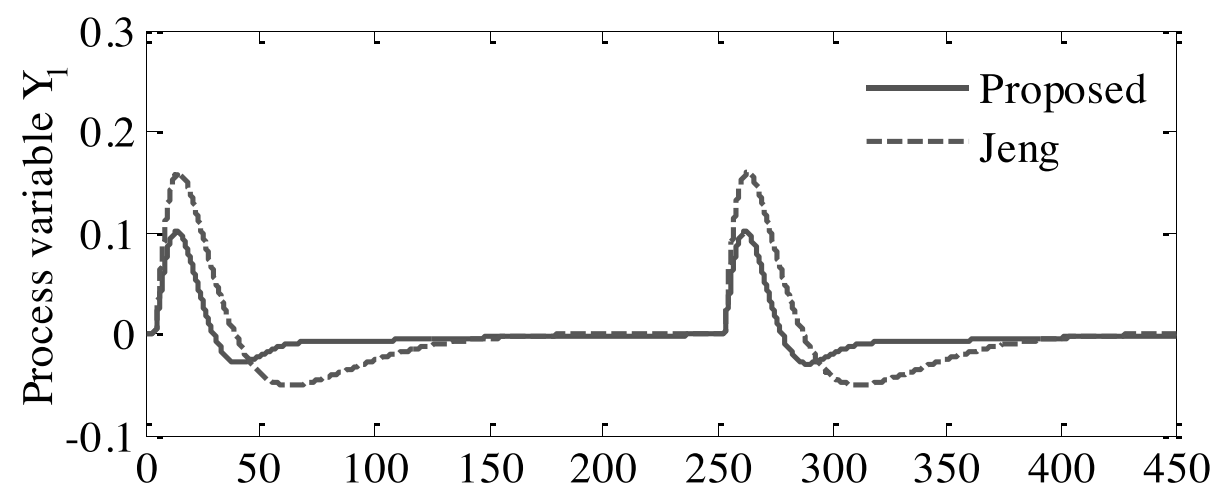

Figure 17. Perturbed response for Example-2 (Case-3).

The figures show that the proposed method is more robust to parametric uncertainty. It is evident from the figures that the present approach, as compared to Jeng (2014), constantly delivers both reduced overshoot and prompt disturbance rejection though holding good servo tracking. The good servo tracking is achieved by using the IMC principle, whereas the improved and prompt disturbance rejection is obtained by modifying the integral time $\tau_{i}=4\left(\tau_{c}+\theta\right)$. The present method also delivers substantial advantage in I.A.E and $\mathrm{y}_{\max }$ value as shown in Table 2. 


\section{Example-3}

The following cascade process studied by Alfaro et al. (2009) is considered against the proposed method.

$G_{p 1}=\frac{e^{-2 s}}{(1+10 s)(1+5 s)}$, and $G_{p 2}=\frac{1}{(1+2 s)(1+0.5 s)}$

The parameters of the primary and secondary controller for the proposed approach and Alfaro et al. (2009) are tabulated in Table 2 (Example 3). In Alfaro et al. (2009), the authors have considered a two-degree-of-freedom (2-DoF) PID controllers for the cascade control structure. In total, four controllers are utilized to control the processes, where two controllers are used in the inner loop and the other two controllers are used in the outer loop. But the proposed scheme utilizes only two controllers.

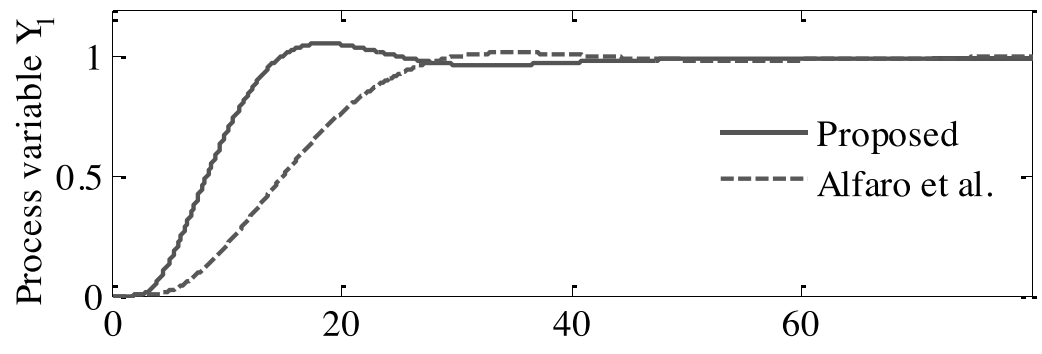

Time $(\mathrm{sec})$

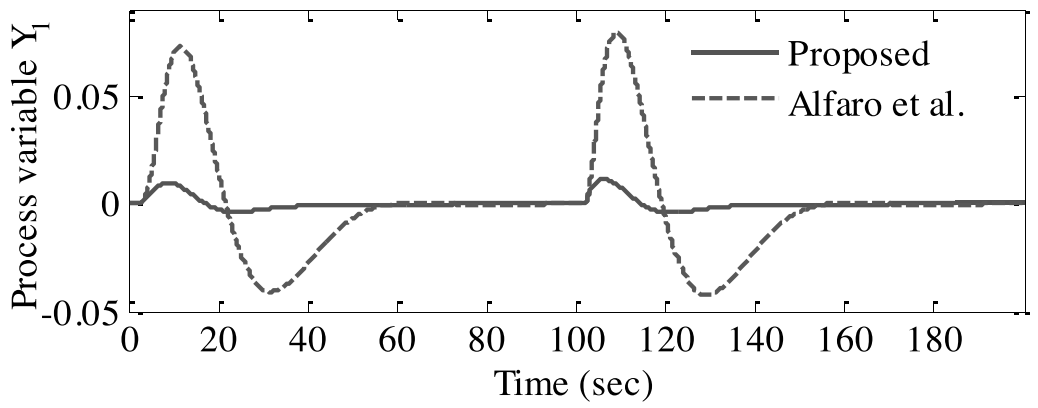

Figure 18. Servo response and regulatory response for Example-3.

With these controllers in the cascade structure, the servo response is generated by applying a unit step set point change (R1) at $\mathrm{t}=0 \mathrm{~s}$, and the regulatory response is generated by introducing a unit step load disturbance as $\mathrm{d} 2=1$ at $\mathrm{t}=0 \mathrm{~s}$ and $\mathrm{d} 1=1$ at $\mathrm{t}=100 \mathrm{~s}$. The servo and regulatory response are shown in Figure 18. The corresponding performance indices are listed in Table 2 (Example-3). Though Alfaro et al. (2009) used four controllers, the performance of the present scheme is superior as indicated in Figure and Table 2 (Example-3).

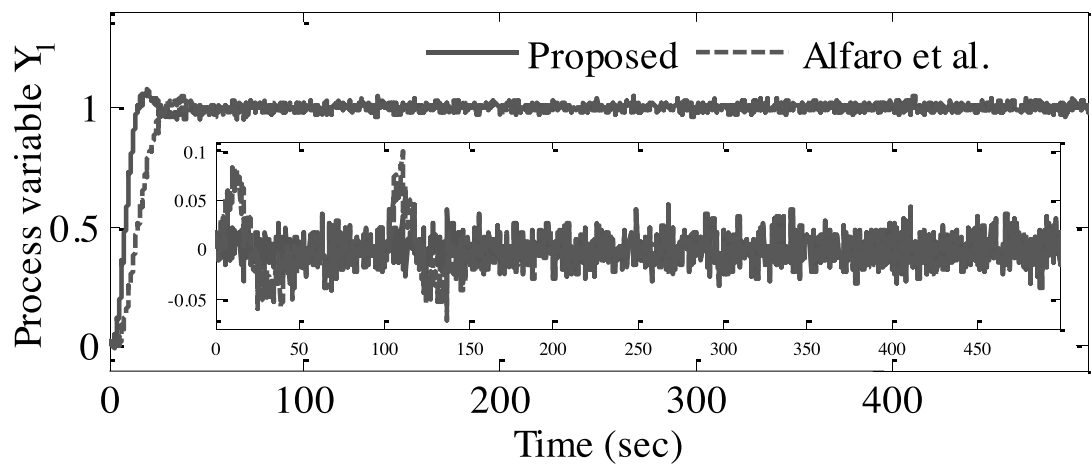

Figure 19. Process variable response due to noise for Example-3. 
Figure 19 illustrates that the present scheme rejects load disturbance and tracks setpoint even in noisy conditions. To analyze the robustness, time delay and gain of the primary plant are changed by $+30 \%$. The perturbed regulatory response is shown in Figure 20. It has been observed from the figure that the design scheme of Alfaro et al. (2009) is more responsive to parameter variations.

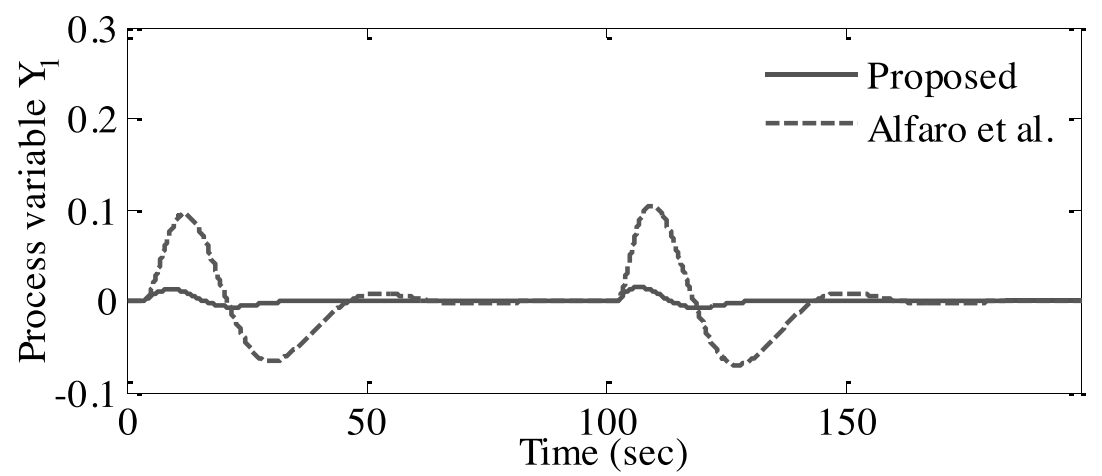

Figure 20. Perturbed response for Example-3.

\section{Example-4}

A second-order primary process and higher-order secondary process are considered as

$$
G_{p 1}=\frac{50}{(0.12 s+1)(0.75 s+1)}, G_{p 2}=\frac{100}{(s+1)(0.001 s+1)^{2}}
$$

For the present scheme, the settings for $G_{c l}$ and $G_{c 2}$ are tabulated in Table 2 (Example 4) and compared with Azar \& Serrano (2014).
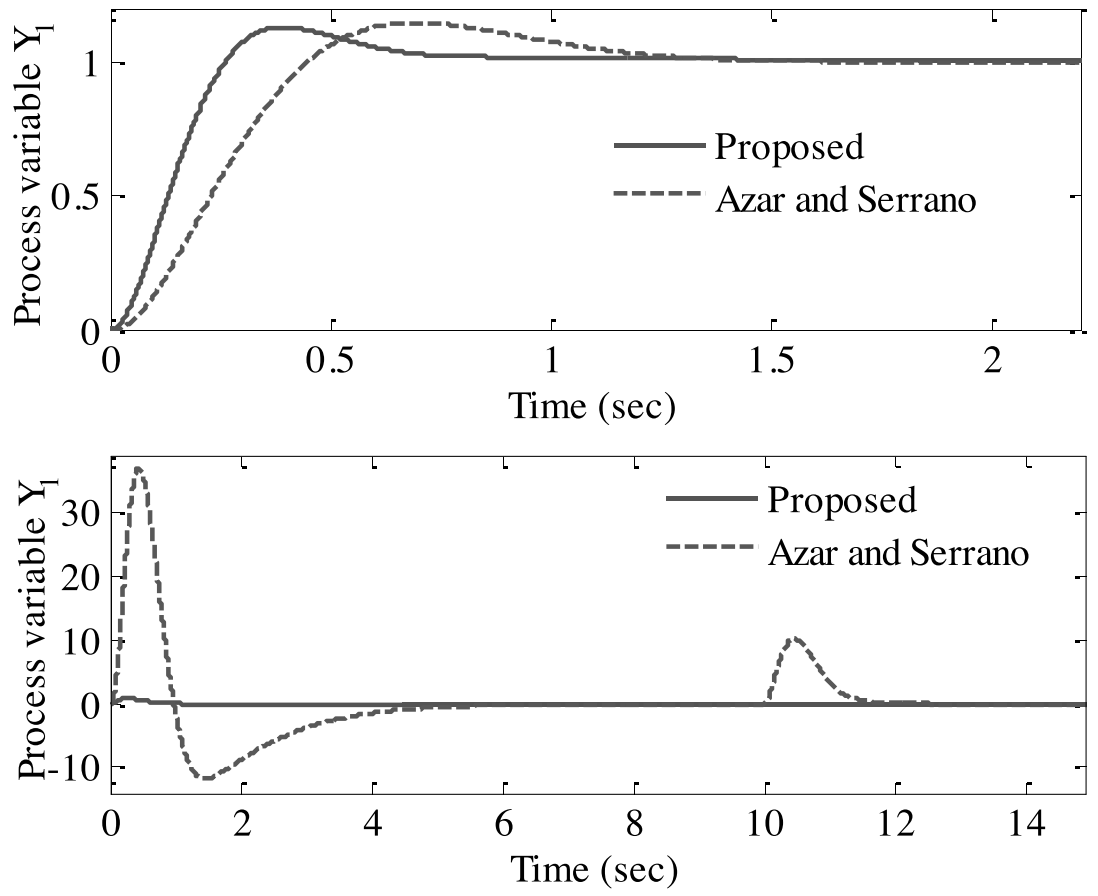

Figure 21. Servo response and regulatory response for Example-4. 
Figure 21 shows the servo response obtained by applying a unit step setpoint change $\left(R_{l}\right)$ at $\mathrm{t}=0 \mathrm{~s}$. The servo response obtained by the present scheme is fast and has a smaller settling time as compared to Azar \& Serrano (2014). The regulatory response shown in Figure 21 is obtained by applying a unit step change as $D_{l}=1$ at $\mathrm{t}=0 \mathrm{~s}$ and $D_{2}=1$ at $\mathrm{t}=10 \mathrm{~s}$, and it is observed that the better load disturbance rejection is obtained by the present scheme. The corresponding performance indices are listed in Table 2 (Example-4). The effect of noise is analyzed in Figure 22.

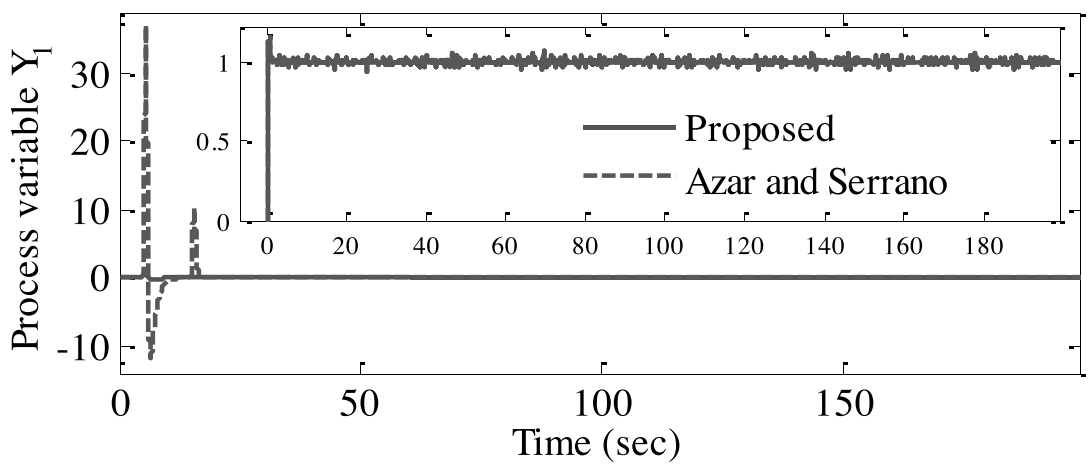

Figure 22. Process variable response due to noise for Example-4.

To analyze robustness, the gains of $G_{p 2}$ and $G_{p l}$ are perturbed by $+30 \%$. The corresponding regulatory response due to uncertainty present in the process is obtained in Figure 23. The figure shows that the proposed method is more robust to parametric uncertainty.

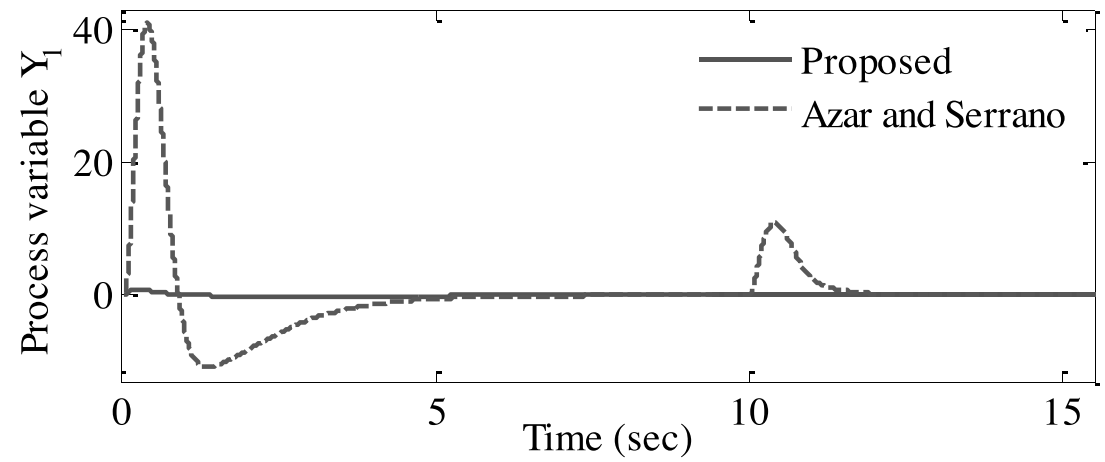

Figure 23. Perturbed response for Example-4.

\section{Example-5}

In this example, the following FOPDT unstable primary process and FOPDT stable secondary process are considered

$$
G_{p 1}=\frac{e^{-2 s}}{(20 s-1)} \text { and } G_{p 1}=\frac{2 e^{-2 s}}{(10 s+1)}
$$

The present scheme is compared against the scheme proposed by Santosh \& Chidambaram (2019). The settings for $G_{c 1}$ and $G_{c 2}$ are tabulated in Table 2 (Example-5). With these controller settings, servo response is generated by applying a unit step setpoint change $\left(R_{l}\right)$ at $\mathrm{t}=0 \mathrm{~s}$, and the regulatory responses are generated by introducing a unit step load disturbance as $d_{2}=1$ at $\mathrm{t}=0 \mathrm{~s}$ and $d_{1}=1$ at $\mathrm{t}=300 \mathrm{~s}$. The corresponding servo and regulatory responses are shown in Figure 24. 

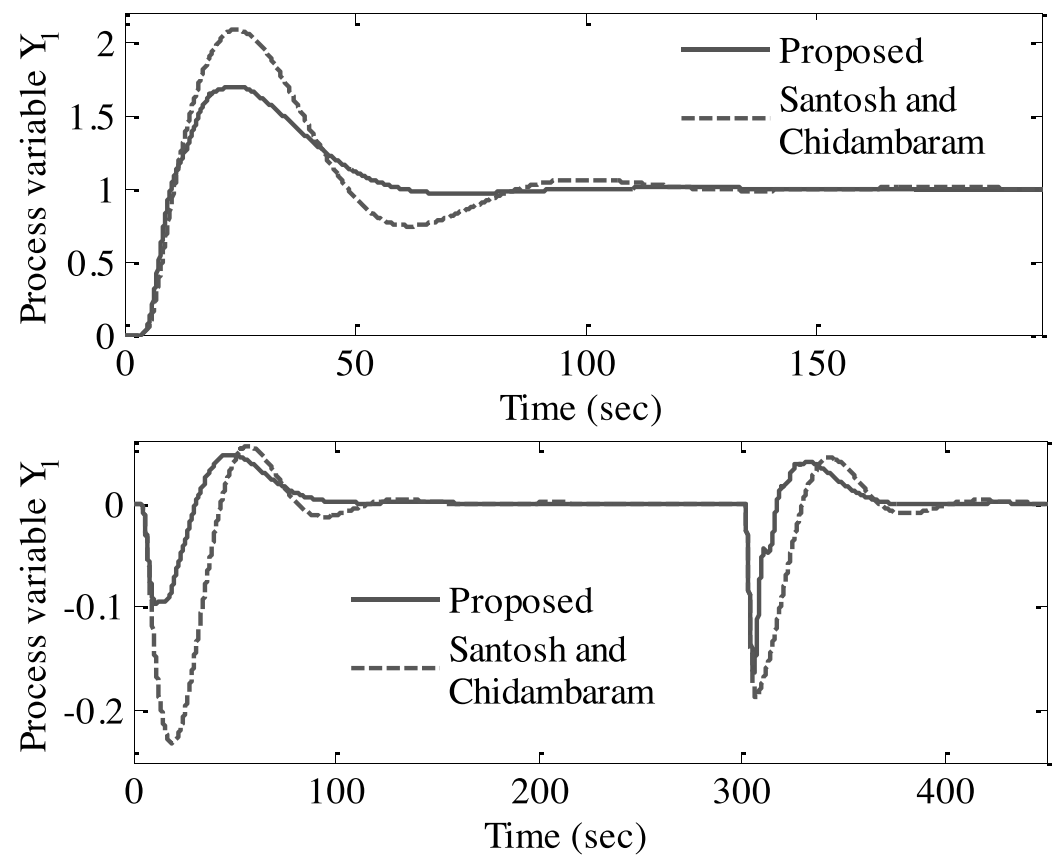

Figure 24. Servo response and regulatory response for Example-5.

The corresponding performance indices are listed in Table 2 (Example-5). From the system response and performance indices, it is noticed that the present scheme can provide better servo tracking and regulatory performances than that of Santosh \& Chidambaram (2019). The effect of noise is analyzed in Figure 25. Figures illustrate that the present scheme rejects load disturbance and tracks setpoint even in noisy conditions. The present scheme is also less responsive to noise than the scheme of Santosh \& Chidambaram (2019) due to the proper selection of time constant $\left(\tau_{c}=\theta\right)$

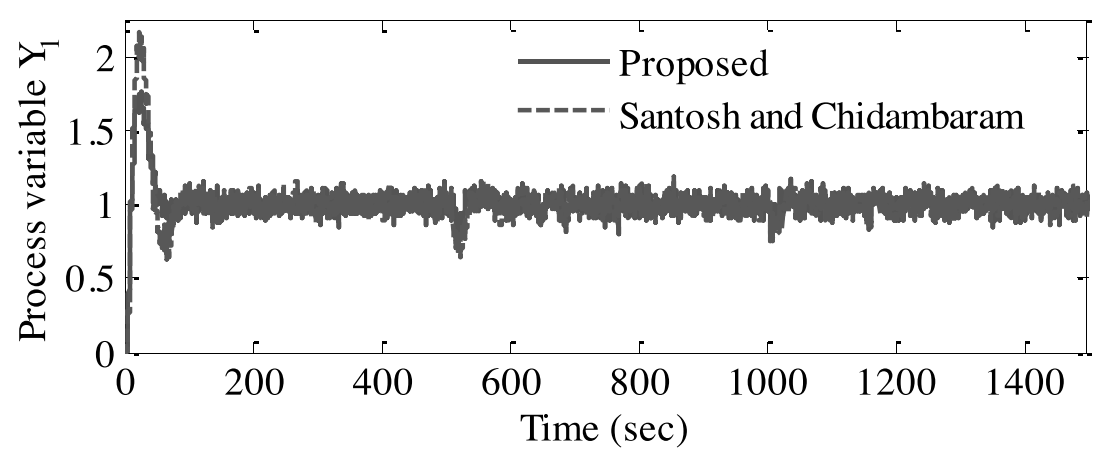

Figure 25. Process variable response due to noise for Example-5.

With the intention to demonstrate the robustness of the proposed scheme, perturbations are introduced in the primary process. The time delay and time constant of the primary plant are changed by $+10 \%$ and $-10 \%$, respectively. The perturbed regulatory response is shown in Figure 26. 


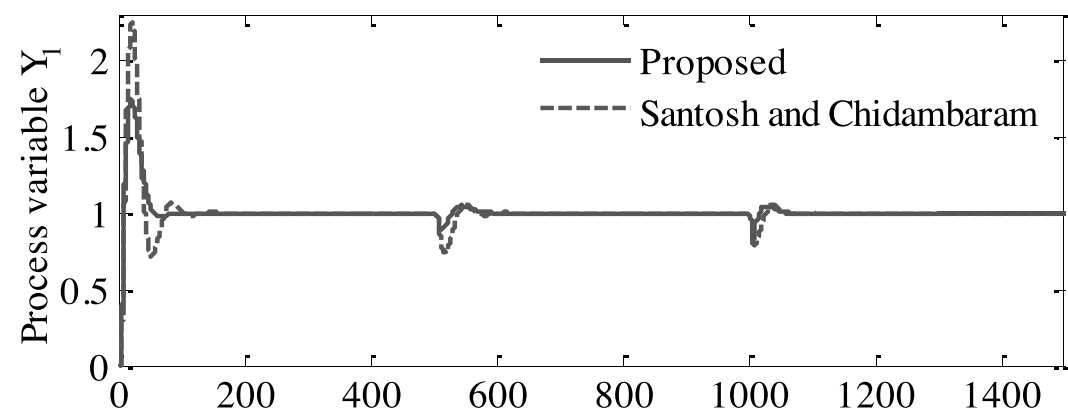

Figure 26. Perturbed response for Example-5.

It has been observed from the figure that the design scheme of Santosh \& Chidambaram (2019) is more responsive to parameter variations, while the proposed method is robust to the parametric uncertainty.

Table 2. Controller settings and evaluation index.

\begin{tabular}{|c|c|c|c|c|c|c|c|c|}
\hline \multirow{2}{*}{$\begin{array}{l}\text { Tuning } \\
\text { Method }\end{array}$} & \multirow{2}{*}{$\begin{array}{l}\text { Secondary } \\
\text { controller }\end{array}$} & \multirow{2}{*}{ Primary controller } & \multicolumn{3}{|c|}{ I.A.E } & \multicolumn{3}{|c|}{$y_{\max }$} \\
\hline & & & $\mathbf{D}_{2}$ & $\mathbf{D}_{1}$ & $\mathbf{R}$ & $\mathbf{R}$ & $\mathbf{D}_{1}$ & $\mathbf{D}_{2}$ \\
\hline \multicolumn{9}{|c|}{ Example-1 } \\
\hline Proposed & $0.85+\frac{6.368}{s}$ & $0.04+\frac{0.12}{s}$ & 0.27 & 0.27 & 7.7 & 1.0 & 0.05 & 0.05 \\
\hline $\begin{array}{l}\text { Kaya and } \\
\text { Nalbantoglu }\end{array}$ & $1.221+\frac{1.221}{s}$ & $1+\frac{0.321}{s}+0.61 s$ & 0.8 & 0.8 & 4.1 & 1.0 & 0.16 & 0.16 \\
\hline \multicolumn{9}{|c|}{ Example-2 } \\
\hline \multicolumn{9}{|c|}{ Case-1 } \\
\hline Proposed & $12.93+\frac{15.00}{s}$ & $2.58+\frac{0.122}{s}+4.64 s$ & 0.03 & 0.03 & 8.5 & 1.03 & 0.002 & 0.002 \\
\hline Jeng & $3.633+\frac{3.042}{s}$ & $1.974+\frac{0.1376}{s}+6.0246 s$ & 0.2 & 0.2 & 10.3 & 1.1 & 0.01 & 0.01 \\
\hline \multicolumn{9}{|c|}{ Case-2 } \\
\hline Proposed & $1.06+\frac{0.065}{s} ; F=0.5$ & $0.075+\frac{9.25 \mathrm{e}-04}{s} ; F=1.25$ & 107 & 36.5 & 135.6 & 1.0 & 0.21 & 0.6 \\
\hline Jeng & $0.617+\frac{0.596}{s}$ & $0.088+\frac{0.0010}{s}+2.273 s$ & 202 & 68.9 & 120 & 1.1 & 0.47 & 1.32 \\
\hline \multicolumn{9}{|c|}{ Case- 3} \\
\hline Proposed & $1.39+\frac{1.15}{s}$ & $0.076+\frac{8.24 \mathrm{e}-04}{s}+0.41 s$ & 5.78 & 6.07 & 249 & 1.25 & 0.2 & 0.2 \\
\hline & $F=0.5$ & $F=0.5$ & & & & & & \\
\hline
\end{tabular}




\begin{tabular}{cccccccccc}
\hline Jeng & $1.366+\frac{1.60}{s}$ & $0.086+\frac{0.0015}{s}+0.732 s$ & 12.5 & 12.5 & 250 & 1.28 & 0.32 & 0.32 \\
\hline Proposed & $8.7356+\frac{3.5802}{s}$ & $2.36+\frac{0.113}{s}+4.57 s$ & 0.17 & 0.16 & 9.55 & 1.05 & 0.01 & 0.009 \\
\hline Alfaro et al. $\quad C_{r 2}=.96+\frac{0.58}{s}$ & $C_{r 1}=0.814+\frac{.094}{s}$ & & & & & & & & \\
& $C_{y 2}=1.166+\frac{0.58}{s}$ & $C_{y 1}=1.27+\frac{0.094}{s}+\frac{2.32 s}{0.18 s+1}$ & 1.60 & 1.56 & 15.8 & 1.02 & 0.08 & 0.07
\end{tabular}

\section{Example-4}

$\begin{array}{lllllllll}\text { Proposed } & 4.61+\frac{2.218}{s} & 0.195+\frac{0.26}{s}+0.0084 s & 0.9 & 0.02 & 0.17 & 1.13 & 0.9 & 0.05\end{array}$

\begin{tabular}{|c|c|c|c|c|c|c|c|c|}
\hline $\begin{array}{l}\text { Azar and } \\
\text { Serrano }\end{array}$ & $0.22+\frac{0.21}{s}+0.0003 s$ & $0.07+\frac{0.129}{s}+0.0029 s$ & 40.5 & 9.24 & 1.8 & 1.14 & 37.1 & 10.3 \\
\hline \multicolumn{9}{|c|}{ Example-5 } \\
\hline Proposed & $2.52+\frac{0.15}{s}+1.8 s$ & $3.26+\frac{0.101}{s}+7.0 s$ & 3.2 & 2.3 & 25.7 & 1.7 & 0.18 & 0.11 \\
\hline $\begin{array}{l}\text { Santosh and } \\
\text { Chidambaram }\end{array}$ & 2.27 & $2.75+\frac{0.103}{s}$ & 6.6 & 4.3 & 39.3 & 2.24 & 0.21 & 0.25 \\
\hline
\end{tabular}

\section{DISCUSSION}

Based on the above results, it is clear the controller settings of the proposed approach are found acceptable with reference to both robustness and performance over a wide range of processes. Furthermore, the proposed method has a distinctive feature that one can select the detuning parameter $(\mathrm{F})$ to achieve i) more robustness $(\mathrm{F}>1)$, ii) fast system response $(\mathrm{F}<1)$, and iii) fast and robust system response $(\mathrm{F}=1)$. The results obtained for Examples 1, 2 (Case-1), 3, 4, and 5 are deprived of the detuning factor $(\mathrm{F}=1)$, while in case of Example 2 (Case-2), the detuning factor is selected as $\mathrm{F}<1$ for inner loop and $\mathrm{F}>1$ for the outer loop. In Example 2 (Case-3), $\mathrm{F}$ is selected as $\mathrm{F}<1$ for both loops.

The present work utilizes the traditional cascade control system and computationally easy approach for tuning the controllers. However, the earlier work follows i) open-loop plant model, ii) complex cascade structure, iii) number tuning of parameters, iv) two or more controllers, and v) computationally difficult approach, which is less preferred in the process industry. Hence, the present work is based on closed-loop plant model based controller design in a simple cascade controller structure. In Azar \& Serrano (2014) and Kaya \& Nalbantoglu (2016), open-loop plant models are used to design the controller in CCS. The primary and secondary processes of Azar \& Serrano (2014) are further approximated into FOPDT and SOPDT by using the Half rule. The approximation of the actual plant is done by using open-loop test and subsequently; further approximation by using half rule results in a less effective controller or less robust controller, whereas the proposed approach acquired process data by closed-loop plant test for controller design and is anticipated to deliver an effective controller. The main merit of the present approach is that it functions properly on a wide range of process models for the process industry. Note that the work of Alfaro et al. (2009); Jeng 
(2014); Azar \& Serrano (2014); Kaya \& Nalbantoglu (2016); and Santosh \& Chidambaram (2019) has already demonstrated supremacy over various prevailing approaches in the literature. Another contribution of the present work is that it follows standard CCS with only two controllers that deliver better setpoint tracking and enhances disturbance rejection in contrast to the recently published literature (Alfaro et al., 2009; Jeng, 2014; Azar \& Serrano, 2014; Kaya \& Nalbantoglu, 2016; Santosh \& Chidambaram, 2019). Note that Alfaro et al. (2009) have used four controllers and Azar \& Serrano (2014) have used six parameters to control process in CCS.

\section{CONCLUSION}

The closed-loop setpoint experiment has been performed on SOPDT, higher-order stable, higher-order IPTD, and unstable primary processes in cascade control configuration to obtain the PI/PID controller settings. In a closed-loop setpoint experiment, P-controller is used to obtain the closed-loop setpoint response. From the response, a certain set of data is obtained. By using this set of data, the controller of the secondary loop and the primary loop are tuned. Simulations have been conducted on five examples to analyze the performance of the proposed method. Simulations show consistently enhanced closed-loop performance of the proposed method over the recently published methods. It is obvious that the better closed-loop performance is a result of good controller tuning. Specifically, for load disturbance, the proposed method shows better performance when compared to the previously cited method. The foremost advantages of the proposed method are as follows.

i) It attenuates the drawbacks of the plant-model mismatch as the controllers are obtained from the set of system input and output.

ii) As the obtained plant data contains more useful and straightforward information about plant dynamics than the open-loop test, the present approach can provide a much effective controller. In addition, improved disturbance rejection is a worthwhile achievement because the CCS is mainly committed to enhance disturbance rejection.

iii) In the presence of noise and perturbation, the proposed method can provide a better and stable closed-loop response.

iv) It functions properly on a wide range of process models for the process industry, comprising FOPDT plus higherorder, integrating, inverse response, and unstable processes.

\section{REFERENCES}

Alfaro, V.M., Vilanova, R. \& Arrieta, O. 2009. Robust tuning of Two-Degree-of-Freedom (2-DoF) PI/PID based cascade control systems. Journal of Process Control, 19:1658-1670.

Anwar, M.N., Siddiqui, M.A., Laskar, S.H. \& Yadav, A. 2019. PIDA Controller Design for Higher Order Stable Process with Inverse Response Characteristic. International Conference on Computational and Characterization Techniques in Engineering and Sciences. India.

Astrom, K.J. \& Hagglund, T. 1995. PID controllers: theory, design, and tuning. Research Triangle Park, Instrument society of America. NC.

Azar, A.T. \& Serrano, F.E. 2014. Robust IMC-PID tuning for cascade control systems with gain and phase margin specifications. Neural Computing and Applications, 25:983-995.

Badgwell, T.A., Pastusek, P.E., Kumaran, K. \& Schmidt, D. 2019. Controller with Automatic Tuning and Method. US Patent Application, 16/133,941.

Barnish, B.R. 1989. A generalization of Kharitonovo's four-polynomial concept for Robust stability problems with linearly dependent coefficient perturbations. IEEE Transaction on Automatic Control, 34(2):157-165.

Bhattacharyya, S.P. \& Keel, L.H. 1995. Robust control: the parametric approach. Advances in control education 1994, Pp. 49-52. Pergamon.

Chen, D. \& Seborg, D.E. 2002. PI/PID controller design based on direct synthesis and disturbance rejection. Industrial \& Engineering Chemistry Research, 41:4807-4822.

Dasari, P.R., Alladi, L., Rao A.S. \& Yoo, C.K. 2016. Enhanced design of cascade control systems for unstable processes with time delay. Journal of Process Control, 45:43-54. 
Jeng, J.C. \& Lee, M.W. 2012. Simultaneous automatic tuning of cascade control systems from closed-loop step response data. Journal of Process Control, 22:1020-1033.

Jeng, J.C. 2014. Simultaneous closed-loop tuning of cascade controllers based directly on setpoint step-response data. Journal of Process Control, 24:652-662.

Jeng, J.C. 2015. Closed-loop tuning of setpoint-weighted proportional-integral-derivative controllers for stable, integrating, and unstable processes: A unified data-based method. Industrial \& Engineering Chemistry Research, 54:1041-1058.

Jeng, J.C. 2015. A model-free direct synthesis method for PI/PID controller design based on disturbance rejection. Chemometrics and Intelligent Laboratory Systems, 147:14-29.

Jeng. J.C. \& Ge, G.P. 2016. Disturbance-rejection-based tuning of proportional-integral-derivative controllers by exploiting closed-loop plant data. ISA Transaction, 62:312-324.

Kaya, İ. \& Nalbantoglu, M. 2016. Simultaneous tuning of cascaded controller design using genetic algorithm. Electrical Engineering, 98:299-305.

Lee, Y., Oh, S. \& Park, S. 2002. Enhanced control with a general cascade control structure. Industrial \& Engineering Chemistry Research, 41:2679-2688.

Leva, A. \& Marinelli, A. 2009. Comparative analysis of some approaches to the autotuning of cascade controls. Industrial \& Engineering Chemistry Research, 48:5708-5718.

Luyben, W.L. 1989. Process modeling, simulation and control for chemical engineers. McGraw-Hill Higher Education.

Luyben, W.L. 2001. Getting more information from relay-feedback tests. Industrial \& Engineering Chemistry Research, 40:43914402.

Padhan, D.G. \& Majhi, S. 2012. An improved parallel cascade control structure for processes with time delay. Journal of Process Control, 22:884-898.

Padhan, D.G. \& Majhi, S. 2013. Enhanced cascade control for a class of integrating processes with time delay. ISA Transaction, 52:45-55.

Raja, G.L. \& Ali, A. 2018. Modified series cascade control strategy for integrating processes. Indian Control Conference, India.

Rivera, D.E., Morari, M. \& Skogestad, S. 1986. Internal model control: PID controller design. Industrial \& Engineering Chemistry Research, 25(1):252-265.

Santosh, S. \& Chidambaram, M. 2019. Enhanced relay autotuning of unstable series cascade systems. Indian Chemical Engineering: 61:1-14.

Seborg, D.E., Mellichamp, D.A., Edgar, T.F. \& Doyle III, F.J. 2010. Process dynamics and control. John Wiley \& Sons.

Shamsuzzoha, M. \& Skogestad, S. 2010. The setpoint overshoot method: A simple and fast closed-loop approach for PID tuning. Journal of Process Control, 20:1220-1234.

Shamsuzzoha, M. 2013. Closed-loop PI/PID controller tuning for stable and integrating process with time delay. Industrial \& Engineering Chemistry Research, 52:12973-12992.

Skogestad, S. 2004. Simple analytic rules for model reduction and PID controller tuning. Model Identification Control, 25:85-120.

Tyreus, B.D. \& Luyben, W.L. 1992. Tuning PI Controllers for Integrator/Dead Time Processes. Industrial \& Engineering Chemistry Research, 31:2625-2628.

Uma, S., Chidambaram, M., Rao A.S. \& Yoo, C.K. 2010. Enhanced control of integrating cascade processes with time delays using modified Smith predictor. Chemical Engineering Science, 65:1065-1075.

Venkatashanker, V. \& Chidambaram, M. 1994. Design of P and PI Controllers for Unstable First-Order plus Time Delay Systems. International Journal of Control, 60:137-144.

Veronesi, M. \& Visioli, A. 2011. Simultaneous closed-loop automatic tuning method for cascade controllers. IET Control Theory Application, 5:263-270.

Yin, C.Q., Wang, H., Sun, Q. \& Zhao, L. 2019. Improved Cascade Control System for a Class of Unstable Processes with Time Delay. International Journal of Control Automation and Systems, 17:126-135.

Zeng, D., Zheng, Y., Luo, W., Hu, Y., Cui, Q., Li, Q. \& Peng, C. 2019. Research on Improved Auto-Tuning of a PID Controller Based on Phase Angle Margin. Energies, 12:1704-1720.

Ziegler, J.G. \& Nichols, N.B. 1942. Optimum settings for automatic controllers. Transaction of ASME, 64: 759-768. 